Cinémas

Revue d'études cinématographiques

Journal of Film Studies

\title{
L'un(e) dort, l'autre pas : la scène de la veille dans les scénarios et quelques romans de Réjean Ducharme
}

\section{Jacqueline Wiswanathan}

Volume 5, numéro 1-2, automne 1994

URI : https://id.erudit.org/iderudit/1001013ar

DOI : https://doi.org/10.7202/1001013ar

Aller au sommaire du numéro

Éditeur(s)

Cinémas

ISSN

1181-6945 (imprimé)

1705-6500 (numérique)

Découvrir la revue

Citer cet article

Wiswanathan, J. (1994). L'un(e) dort, l'autre pas : la scène de la veille dans les scénarios et quelques romans de Réjean Ducharme. Cinémas, 5(1-2), 189-209. https://doi.org/10.7202/1001013ar
Résumé de l'article

Les séquences finales des scénarios de Ducharme présentent une similitude frappante : chacune réunit un personnage éveillé et un personnage endormi. Dans cet article, nous analysons la signification de cette scène emblématique que nous rapprochons ensuite de scènes semblables dans l'oeuvre romanesque. Si la scène de la veille occupe une place aussi importante dans les scénarios, c'est qu'en plus de concrétiser des rapports affectifs fondamentaux entre les personnages, elle se prête particulièrement à une représentation cinématogtaphique. Par cette analyse, nous espérons démontrer que le scénario est, tout autant que le roman ou le théâtre, profondément révélateur du monde imaginaire d'un écrivain. 


\title{
L'un(e) dort, l'autre pas: la scène de la veille dans les scénarios et quelques romans de Réjean Ducharme ${ }^{1}$
}

\section{Jacqueline Viswanathan}

\begin{abstract}
RÉSUMÉ
Les séquences finales des scénarios de Ducharme présentent une similitude frappante: chacune réunit un personnage éveillé et un personnage endormi. Dans cet article, nous analysons la signification de cette scène emblématique que nous rapprochons ensuite de scènes semblables dans l'œuvre romanesque. Si la scène de la veille occupe une place aussi importante dans les scénarios, c'est qu'en plus de concrétiser des rapports affectifs fondamentaux entre les personnages, elle se prête particulièrement à une représentation cinématographique. Par cette analyse, nous espérons démontrer que le scénario est, tout autant que le roman ou le théâtre, profondément révélateur du monde imaginaire d'un écrivain.
\end{abstract}

\section{ABSTRACT}

The final sequences of Ducharme's screenplays are strikingly similar: they bring together two characters, one is awake while the other is asleep. In this article, we analyze the meaning of this emblematic scene in terms of relationships between the characters. We then relate this particular sequence to similar passages in the novels. We thus hope to show that screenplays can be just as revealing of a writer's imaginary world as fiction or drama. 
Le scénario entre cinéma et roman : état présent de la critique

Est-il besoin de le répéter? Le cinéma et la littérature sont profondément liés dans l'imaginaire contemporain. Si le cinéma se nourrit voracement de romans, comme en témoigne le nombre incalculable d'adaptations filmées, il a aussi influencé la vision moderne de la réalité et sa représentation romanesque. Au stade de la création aussi, les liens sont multiples: nombre de romanciers contemporains sont scénaristes. Au Québec, on peut citer, entre autres, Hubert Aquin, Jacques Benoît, Marie-Claire Blais, Jacques Godbout, Anne Hébert, André Major, Jacques Savoie, Yves Thériault.

La toute grande majorité des études critiques qui tentent de jeter un pont entre littérature et cinéma traite d'adaptations filmées de romans. Si cette approche a le mérite d'explorer le domaine le plus évident d'une rencontre entre ces deux médias, elle a cependant le désavantage de présenter le film comme une sorte de sous-produit, tout en ignorant parfois l'évidence: le film, même tiré d'un roman, est une œuvre à part entière et l'adaptateur n'a pas forcément à être fidèle au texte d'origine. Une autre approche consiste à comparer la forme et les techniques cinématographiques et romanesques, notamment du point de vue de l'organisation du récit et de la focalisation. On peut citer quelques travaux fondamentaux dans ce domaine: L'CEilcaméra de François Jost, Récit filmique, récit romanesque de Francis Vanoye et Film and Fiction de Keith Cohen. Cette étude comparative peut mener à la démonstration de rapports d'influence du cinéma sur la littérature comme dans l'ouvrage bien connu de Claude-Edmonde Magny, L'Âge du roman américain ou dans de nombreuses études sur le Nouveau Roman. En revanche, on peut démontrer l'influence de la littérature sur le cinéma, comme dans l'étude de Marie-Claire RoparsWuilleumier.

Rares sont les analyses qui abordent la question de la coexistence et souvent de l'interpénétration des deux médias au sein de l'œuvre d'un même écrivain. Le livre de Jeanne-Marie Clerc, Écrivains et cinéma, présente une étude de ce phénomène transmédiatique et montre bien à quel point le cinéma a transformé la vision même du monde chez beaucoup de romanciers. Il ne 
s'agit pas seulement de la forme comme avait voulu le montrer Claude-Edmonde Magny, mais de la conception même du rapport entre les mots, les images et les choses. Jeanne-Marie Clerc analyse aussi, fait assez exceptionnel dans la critique comparée de la littérature et du cinéma, les scénarios de certains écrivains connus (Sartre, Malraux, Cocteau, Giono, Duras, etc.) pour y découvrir la profonde influence de la pratique d'une écriture cinématographique sur la totalité de l'œuvre.

Écrivains et cinéma est un des rares ouvrages qui, prenant le scénario au sérieux, ne le considère pas avant tout comme un instrument de travail. La critique de scénario, si elle veut être autre chose qu'un outil didactique (comme dans les nombreux manuels d'enseignement du scénario) doit poursuivre cette recherche et élaborer des schémas d'analyse qui reconnaissent les particularités d'un texte destiné à s'effacer devant le film qui est sa raison d'être. Elle doit aussi tenir compte de nombreux aspects communs entre le scénario et d'autres textes fictionnels comme, par exemple, la narrativité, la construction des personnages et le système de leurs rapports, l'exploitation du dialogue et l'alternance entre parties narrativo-descriptives et parties dialoguées, le code des connotations culturelles, l'intertextualité, etc. Une telle analyse pourrait démontrer que le scénario peut être une œuvre aussi richement révélatrice de l'imaginaire d'un écrivain que le roman et le théâtre ${ }^{2}$. C'est le but de la présente étude.

\section{Ducharme scénariste}

Réjean Ducharme s'est toujours refusé à adapter ses romans. C'est ainsi qu'il a plus ou moins désavoué la version cinématographique du Nez qui voque ${ }^{3}$. Cependant, comme nombre d'autres romanciers contemporains, il est l'auteur de plusieurs scénarios. Les Bons Débarras, écrit en 1977, remanié avec Francis Mankiewicz en 1979, a été réalisé par ce dernier et distribué en 1980. Primé au festival de Berlin, ce film a été très bien accueilli. Ce succès a rendu possible la réalisation, également par Francis Mankiewicz, des Beaux Souvenirs (écrit en 1980, remanié et réalisé en 1981). Ducharme est aussi l'auteur de deux scénarios inédits et non réalisés: Comme tu dis et Le Bonheur des 


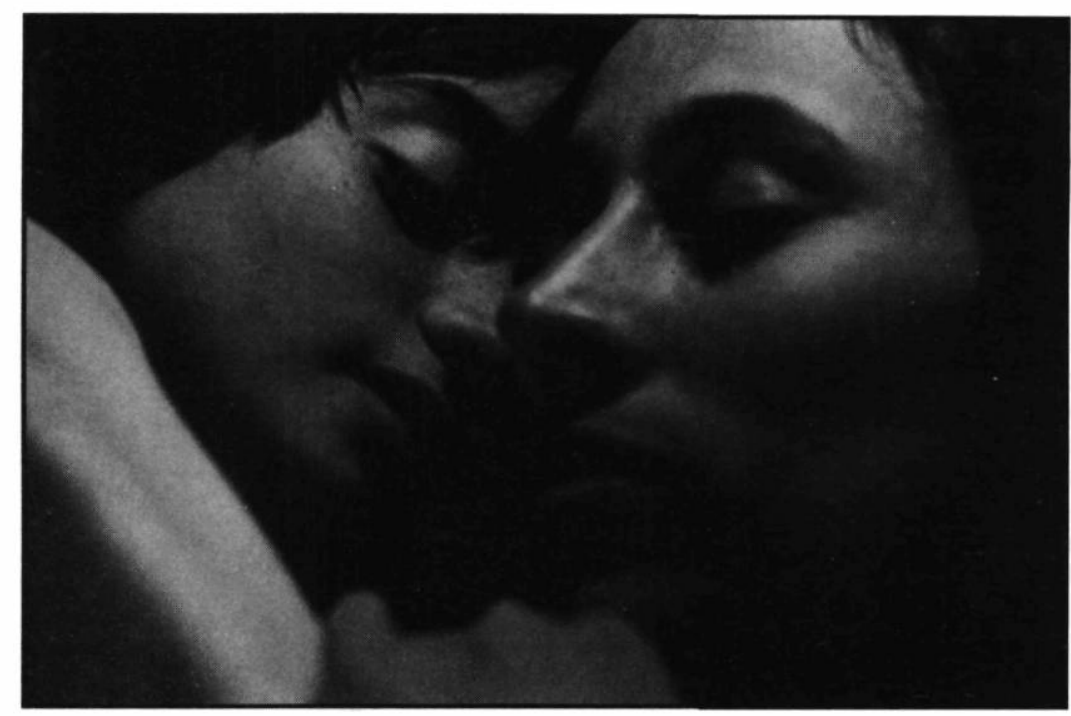

\section{Les Bon Débarras de Francis Mankiewicz (1980)}

Collection Cinémathèque québécoise

autres dont les tapuscrits sont également conservés à la Cinémathèque québécoise ${ }^{4}$. Le texte de Comme tu dis contient une lettre de Ducharme à Jean-Claude Labrecque datée de 1978. Le Bonheur des autres est le plus récent des scénarios (probablement 1988). Il était également destiné à Francis Mankiewicz.

Il existe peut-être d'autres scénarios, mais pas à ma connaissance. Le succès très mitigé des Beaux Souvenirs a sans doute découragé les producteurs. Cependant, il est permis d'espérer que ces œuvres extrêmement originales et valables d'un point de vue cinématographique ne resteront pas lettre morte. L'évolution de l'industrie médiatique - multiplication des chaînes de télévision, production de films destinés à une diffusion en cassettes vidéo - encouragera peut-être la production d'œuvres "non commerciales", comme les films réalisés à partir de scénarios de Ducharme. Peut-être la prise en compte, par la critique "littéraire", de cette partie de l'œuvre d'un des plus grands écrivains québécois pourra-t-elle même contribuer à sa diffusion ${ }^{5}$.

Cette étude aborde un aspect très particulier des scénarios de Réjean Ducharme: la récurrence d'une scène emblématique dont nous tenterons d'analyser la signification. Nous espérons 
ainsi caractériser certains aspects profondément révélateurs du monde fictif, propre à l'imaginaire de cet auteur. Nous voulons aussi rapprocher les scénarios et les romans, tout en reconnaissant l'importance des caractéristiques propres à chaque genre.

\section{Structures archétypiques et mythes personnels}

Plusieurs critiques bien connus ont déjà réussi à caractériser l'univers imaginaire d'une œuvre. Je pense ici aux études de Jean-Pierre Richard, à la critique de Georges Poulet, qui analyse, par exemple, à travers l'expérience existentielle du temps et de l'espace, la vision particulière d'un écrivain. Je pense surtout aux réseaux associatifs, aux mythes personnels mis au jour par Charles Mauron, qui superpose les textes d'un même auteur pour y révéler des métaphores obsédantes, des structures archétypiques. Mauron a analysé plusieurs œuvres dramatiques mais, et ceci n'étonnera pas, jamais de scénarios. Ce type d'analyse n'a d'ailleurs jamais été utilisé à propos des romans et des scénarios d'un même auteur. C'est qu'on voit souvent le scénario comme un texte utilitaire, sans réelle valeur artistique et aussi, sans doute, comme un texte qui ne reflète pas aussi profondément que d'autres l'imaginaire d'un créateur. La méthode de Mauron mérite qu'on s'y attarde. Elle me paraît riche en possibilités d'applications à l'analyse des scénarios.

La première étape de la démarche du père de la psychocritique s'appuie sur l'observation empirique: elle correspond à la recherche des "éléments obsédants" réapparaissant à travers plusieurs œuvres d'un même auteur. Pour la poésie, il s'agit surtout d'analogies verbales associées à des images, des comparaisons ou des métaphores. Dans le cas du théâtre, il s'agit plutôt de figures et de situations. Chez Molière, par exemple, Mauron croit détecter la "dominante obsessive" du conflit entre les générations et de la tromperie féminine, liés au motif du voleur volé. Il rapporte ensuite ces "structures affectives personnelles" aux grands mythes archétypiques comme l'CEdipe. Tout en m'inspirant de cette approche, je voudrais l'adapter au type particulier de discours que présente le scénario. 


\section{La scène de la veille}

Je partirai donc de cette observation: il est une similitude frappante entre les séquences finales des scénarios dont les synopsis se trouvent en appendice de cet article. Dans le scénario Les Bons Débarras, la fin du film est ainsi décrite par Ducharme :

\section{INT. NUIT Chambre Michelle.}

Manon lit, à sa mère épuisée, pratiquement endormie, le dernier paragraphe des Hauts de Hurlevent. Elle s'étend de tout son long sur sa mère, la prend par le cou, la réveille encore.

\section{MANON}

Maman...

MICHELLE (rouvrant les yeux avec peine)

Quoi, mon amour?

\section{MANON}

J'ai fini (faisant une bise à Michelle). Bonne nuit.

L'image gèle: celle de Manon seule avec Michelle dans son île imaginaire et qui s'est allongée sur elle, dans le halo de phare de sa lampe de poche, comme pour inaugurer son règne (p. 228, séq. 141).

La séquence finale du scénario des Beaux Souvenirs se présente ainsi :

Papa est sur le lit de Marie. Ses bras et ses jambes tremblent. Marie parle et agit avec une mesure qui ressemble à la plus froide fermeté... Elle roule Papa dans l'imperméable qu'elle a étendu à côté de lui et elle l'enferme dedans, en le lui boutonnant dans le dos, avec les bras le long du corps. Papa s'endort (p. 104, séq. 71).

Comme tu dis se termine par une séquence comparable:

Il passe devant la chambre de Sabine. Il regarde par les rideaux entrouverts... Allongée sur un oreiller, comme un bébé: une poupée de garçon, une sorte de Barbie au masculin. Benoît lâche un soupir. Un soupir d'homme encore plus à plaindre que tout à l'heure... Et son regard se fige sur la poupée, couchée dans le mou de l'oreiller (p. 49, séq. 40). 
Quand j'ai enfin pu lire le dernier scénario de Ducharme, j'ai été frappée de constater qu'il se clôturait ainsi :

Elle pousse la porte. L'image du petit et de sa gardienne enlacés dans un profond sommeil lui refait faire son beau sourire. Puis, comme on dit, la face lui tombe: l'étreinte de Violette (qui nous rappelle celle de la Scène 21) a quelque chose de pas normal, de pas beau. Le bébé est devenu une "poupée froide et molle". (p. 68, séq. 80)

Les récurrences entre séquences finales pourraient se formuler ainsi : chacune est une scène de veille, c'est à dire qu'elles réunissent toutes un personnage éveillé et un personnage endormi, avec ces curieuses variantes: le "gisant" est une poupée dans Comme tu dis alors que dans Le Bonheur des autres, l'un des deux personnages "endormis" est "comme une poupée froide et molle». L'importance de la scène de la veille est aussi attestée par la présence de séquences semblables qui la préfigurent. Les Bons Débarras ne contient pas moins de six séquences de coucher ou de veille. On en trouve quatre dans Les Beaux Souvenirs et dans Comme tu dis. Ducharme lui-même, dans le scénario du Bonheur des autres, rappelle, dans la scène 81 , une autre scène de veille qui l'avait précédée (la séquence 21 ):

Ce que $\mathrm{M}^{\mathrm{me}}$ Degrandpré, qui arrive de veiller, trouve émouvant, puis comique, et qui lui fait faire signe à son mari de monter vite, c'est le ménage à trois: Sébastien qui tourne le dos à Violette qui s'est endormie avec le petit ours en peluche dans les bras. Mais le plus drôle c'est que Violette dort si dur, et si contractée, que M. Degrandpré, qui veut la réveiller en lui jouant le tour de lui ôter l'ourson, se voit obligé de le lui arracher...

\section{L'un(e) dort, l'autre pas : essai d'interprétation}

A partir de ces séquences, privilégiées par leur position en clôture, il s'agira d'abord de définir la configuration des rapports entre personnages nous permettant de caractériser le "roman familial " propre à l'imaginaire ducharmien.

La "superposition" des dernières scènes des Bons Débarras (dorénavant B.D. dans le texte) et des Beaux Souvenirs (B.S.) est 
aisée. Les personnages endormis, la mère pour Manon, le père pour Marie, se trouvent complètement à la merci de l'amour possessif de leurs "petites filles". La scène finale marque la victoire de Manon et de Marie, victoire d'autant plus totale qu'elles ont éliminé leurs rivaux dans le cœur de leurs parents respectifs par des suicides qu'elles ont en partie provoqués: celui de TiGuy, frère de Michelle (B.D.) et celui de Viviane, sœur de Marie (B.S.). Il faut noter l'absence, importante pour le "roman familial ", du père de Manon et de la mère de Marie, absence indispensable à la possession exclusive du parent aimé. La scène de la veille se présente donc comme l'aboutissement de la Quête du Sujet qui possède l'objet désiré grâce à l'élimination des Opposants. Le sommeil des uns permet l'emprise des autres.

Dans la séquence finale de Comme tu dis (C.T.D.), on peut supposer que "la poupée de garçon", cette étrange "Barbie au masculin", couchée par Sabine sur l'oreiller, est une effigie du père, endormi comme dans les autres séquences. Comme dans les autres séquences, la mort rôde derrière le sommeil. Sabine n'est pas là pour contempler sa victoire. Le message qu'elle a laissé sur l'oreiller, "Tu ne me feras plus la mort. / Je n'aurai plus jamais mal au ventre. ", indique qu'elle s'est suicidée. Sabine est, à la différence de Manon et de Marie, un personnage faible ${ }^{6}$. Pour elle comme pour Viviane et Ti-Guy, la seule voie, c'est la mort. Sa victoire sur son père consiste à se libérer de l'emprise affective de celui qui n'a cessé, pendant tout le film, de menacer de se suicider. Elle montre à son père, comme Chateaugué à Mille Milles, un courage qu'il n'aura pas.

Le Bonheur des autres (B.A.) présente une autre variante de la scène de la veille. La Quête principale est ici aussi la recherche de la possession / domination d'un être aimé. La dernière séquence, d'abord euphorique, montre Nicole contemplant deux personnages endormis: son bébé et Violette, la gardienne qu'elle a réussi à " séduire". Cette scène marque donc sa victoire dans la lutte qui l'a opposée à la mère adoptive de Violette: elle les possède tous deux sous son regard. Mais brusquement, le ton change. Nicole comprend que le bébé est mort. D'autre part, Violette aussi lui échappe. La mort du bébé la rend folle et la met complètement à la merci de sa "bienfaitrice". 
Le tableau ci-dessous permet de schématiser les rapports entre personnages figurant dans la scène de la veille:

\begin{tabular}{|l|l|l|}
\hline $\begin{array}{l}\text { Personnages } \\
\text { endormis } \\
\text { soumis }\end{array}$ & $\begin{array}{l}\text { Personnages } \\
\text { éveillés } \\
\text { victorieux }\end{array}$ & $\begin{array}{l}\text { Personnages } \\
\text { absents } \\
\text { rivaux }\end{array}$ \\
\hline $\begin{array}{l}\text { Michelle, la mère } \\
(B . D .)\end{array}$ & Manon & $\begin{array}{l}\text { Ti-Guy (suicide) } \\
\text { le père de Manon }\end{array}$ \\
\hline $\begin{array}{l}\text { Papa }(B . S .) \\
\text { effigie du père (C.T.D.) }\end{array}$ & $\begin{array}{l}\text { (Sabine) } \\
\text { (absente/ suicide) }\end{array}$ & Sabine \\
\hline $\begin{array}{l}\text { Bébé (poupée) } \\
\text { Violette }(B . A .)\end{array}$ & $\begin{array}{l}\text { Nicole } \\
\text { épilogue: Matante } \\
\text { (mère adoptive) }\end{array}$ & $\begin{array}{l}\text { Matante } \\
\text { Nicole }\end{array}$ \\
\hline
\end{tabular}

On constate donc que la mise en scène de personnages endormis, veillés par d'autres personnages, est profondément significative de l'imaginaire ducharmien tel qu'il se concrétise dans les scénarios. La signification du sommeil et de la veille, leur rapport avec la lutte pour l'amour et le pouvoir sont d'ailleurs rendus tout à fait explicites dans ce passage tiré des Beaux Souvenirs:

Les yeux barbouillés et gonflés par sa crise de larmes, Viviane commence à être gagnée par le sommeil. Marie est assise sur le bord du lit et elle la veille, en silence. Et elle l'aime avec ce mélange "critique" de pitié et de cruauté qui est le seul amour qu'elle peut avoir (p. 93, séq. 61).

Par ailleurs, le sommeil est étroitement lié avec la mort, même dans les scènes en apparence paisibles et euphoriques. Ainsi, dans les B.D., Manon, au chevet de sa mère endormie, lit ce passage décrivant le cimetière des Hauts de Hurlevent: "Je me demandais comment quelqu'un pouvait imaginer que ceux qui dormaient dans cette terre tranquille eussent un sommeil troublé.” (B.D., p. 227) À la fin des Beaux Souvenirs, Papa ne dort

L'un(e) dort, l'autre pas : la scène de la veille dans les scénarios et quelques romans... 
pas du sommeil du juste: il sort d'une affreuse crise d'overdose et l'on vient de voir la séquence du suicide de Viviane. Quant à Manon, elle vient de répondre au coup de téléphone annonçant l'accident de Ti-Guy.

Dans les deux premiers scénarios, il importe, comme dans les contes de fées, de réveiller le "mort» endormi pour lui rendre la vie: "Manon s'étend de tout son long sur sa mère, la prend par le cou, la réveille encore" (B.D., p. 227). Dans Les Beaux Souvenirs: "L'andante ma adagio est une grande et pure rêverie... et c'est ainsi que Marie réveille Papa comme s'il était Blanche-Neige ou la Belle au Bois dormant" (B.S., p. 110). Les enfants s'emparent ainsi du pouvoir ultime, celui de donner la vie et le retournement du rapport parent-enfant est accompli: "Marie se fait une sorte de caresse de froid et de mort... Mais c'est aussi comme si elle avait eu une crampe de parturiante" (B.S., p. 110).

La mort de Sabine à la fin de Comme tu dis et la folie de Violette à la fin du Bonheur des autres clôturent les scénarios sur un mode tragique. Le rire niais de Violette, "retombée en enfance", la Barbie masculine dans C.T.D. introduisent aussi une note grinçante. Un remaniement de ces deux scénarios non réalisés, cette fois en collaboration avec le metteur en scène, les aurait peut-être modifiés pour y introduire un happy end, de rigueur au cinéma.

\section{Scénarios et romans}

Quand on compare romans et scénarios de Ducharme, le contraste est frappant: les romans sont pétris de jeux de langage, feux d'artifice d'une créativité indissolublement liée à la forme de l'expression. En revanche, les scénarios, par leur style pragmatique et dépouillé, se plient aux impératifs du genre. Les romans de Ducharme ne privilégient pas de la même façon le mode de présentation scénique qui est fondamental pour tout scénario. Cependant, en dépit de ces différences de style évidentes, on peut retrouver, dans les romans, des scènes de veille qui rappellent les scénarios.

Il me paraît aussi possible de rapprocher certains romans de certains scénarios. Ainsi, je vois une affinité particulière entre L'Avalée des avalés et Les Bons Débarras: Manon est aussi féroce 
que Bérénice, féroce d'intelligence, de cruauté et de soif d'amour. Est-ce coïncidence si Les B.D. se passent dans un village nommé "Val des Vals"? Les paroles de Bérénice contemplant sa mère assoupie reprennent, comme un écho, les éléments caractéristiques de la scène de la veille, y compris la poupée:

\begin{abstract}
Allongée là, tout près dans mon lit, elle me donne l'impression de se laisser appartenir, de me laisser la posséder. Couchée à la place des poupées que j'ai eues, elle me donne l'impression d'être une poupée, d'être toute à moi (1966, p. 106).
\end{abstract}

On y retrouve tous les éléments caractéristiques de la scène de la veille, y compris la substitution poupée / parent. L'enfant tient enfin sa mère emprisonnée dans son sommeil, totalement subjuguée à son amour, un amour dévorant et destructeur. On connaît le pouvoir maléfique de Bérénice comme on sait que Manon des B.D. n'est pas étrangère à la mort de son frère-rival.

On peut rapprocher la dernière scène de la veille des Beaux Souvenirs de ce passage de L'Hiver de force:

Catherine dort si fort, cramponnée à sa fiole de valium que, jusqu'à ce qu'une mouche chatouille sa bouche, nous l'avons crue morte. Comme perdue, comme tombée du ciel dans cette verdure où ses membres nus sont presque engloutis. Catherine dort, toute belle, toute blanche... elle laisse en pleine confiance, en total abandon, la vie marcher toute seule, se penser elle-même. Catherine fait comme les arbres: elle dort. On fait comme les deux seuls nuages du firmament: on la regarde sans rien dire (1973, p. 268).

Comme Les Beaux Souvenirs, L'Hiver de force se passe en partie dans une île pendant l'été. Même si Catherine n'est pas la mère d'André et de Nicole, quel que soit leur rapport familial réel ou figuré, il importe davantage que le personnage endormi soit un être passionnément aimé et désiré. Ce passage du roman établit aussi un rapport entre sommeil et mort car, comme Papa, la Toune s'est droguée. Nicole et André couvrent leur star bienaimée de fleurs, comme une dépouille mortelle, tandis que Marie a roulé Papa dans son imperméable, camisole de force 
mais aussi linceul. Il est également un curieux parallèle, évocateur de mort, entre l'araignée que Marie pose sur le visage de Papa et la grenouille qu'André fait marcher sur la face de Catherine. Au jeu, se mêle une sorte de magie noire: ce geste étrange brise le sort qui maintenait les personnages dans leur sommeil et les ramène à la vie. Il faut pourtant noter une différence importante entre scénarios et romans. Quand Catherine se réveille, elle détruit l'euphorie du moment. Elle échappe à l'amour de Nicole et d'André, s'enfuit de l'île en leur laissant seulement une lettre de rupture. En revanche, Les Beaux Souvenirs prolonge indéfiniment la béatitude de Marie à travers l'adagio du concerto pour basson de Mozart qui accompagne la fin du film: "On regarde un peu pour être sûr qu'il est bien vivant. Il l'est. Et il aime bien sur la face de Marie cette envie de rire d'enfant qui a joué un bon tour" (p. 111, séq. 83). De même, Les Bons Débarras suspend pour l'éternité le bonheur de la possession en "gelant" la dernière image qui immobilise Manon veillant sa mère. En revanche, pour Bérénice, il ne s'agit que d'un moment très fugitif.

On pourrait aussi rapprocher Comme tu dis et $\mathrm{Le} \mathrm{Nez} q u i$ voque. Mille Milles regarde Chateaugué endormie :

Elle dort depuis une heure. Depuis une heure, je la regarde dormir, être jolie, être mon amie, être revenue, être menue, être méprisée par moi. Tous les enfants sont beaux. Tout ce qu'on aime est beau. Tout ce qu'on aime est agréable à regarder (1967, p. 176).

Mais cet attendrissement est de courte durée. À cette scène fait pendant l'horrible fin du roman quand Mille Milles découvre Chateaugué allongée sur le lit, habillée dans sa robe de mariée, baignant dans le sang, endormie pour l'éternité. Chateaugué et Sabine se ressemblent. Par leur suicide, elles ont accompli l'acte que ni Mille Milles ni Benoît n'ont eu le courage de commettre, tout au plus ont-ils abusé de cette menace pour tenir les deux jeunes filles à leur merci.

Dévadé, comme Le Bonheur des autres (les autres titres suggérés par Ducharme sont "Les mauvais services" ou "L'amour et la charité "), met en relief un aspect de l'amour qui était déjà 
inscrit en creux dans toutes les autres œuvres. Bottom et Violette sont des "évadés" de prison. Tous deux ont été recueillis par des patronnes-bienfaitrices. Le lien complexe et parfois destructeur entre amour et charité, amour et dévouement, amour et domination ou soumission est au cœur des rapports entre ces personnages.

Dans le dernier roman de Ducharme, la scène de la veille met en scène le couple fraternel (ici, Bottom et sa sœur Lucie) qui occupe une place privilégiée dans son œuvre:

Je l'expose au fantasme où ma puberté se pâmait. J'avais mon nid dans ses boucles blondes et j'attendais qu'elle s'endorme pour l'envahir, jouir de son trop joli visage. Je trottinais sur ses paupières, m'introduisais dans ses oreilles. Je m'enivrais à la goutte de sueur qu'un mauvais rêve faisait perler sur sa tempe (1990, p. 49).

Ce passage, d'une beauté presque inquiétante, désigne explicitement la valeur fantasmatique de la scène de la veille et décrit merveilleusement bien le rapport entre endormir et séduire, ce pouvoir érotique et subjugant du sommeil, qui le caractérise dans l'imaginaire ducharmien.

Il me semble donc pouvoir affirmer qu'endormir un être aimé, le contempler quand il/elle s'est abandonné au sommeil et connaître par là un moment d'euphorie, venant d'une impression fugitive de possession, est une scène récurrente, emblématique à la fois dans les romans et les scénarios qui démontrent ainsi leur appartenance à un imaginaire commun. Elle correspond à ce besoin d'aimer et aussi souvent de dominer qui consume tous les personnages. La perte de la conscience chez l'autre amène un moment de répit presque toujours illusoire dans une situation souvent porteuse de mort.

La séquence de la veille n'est évidemment pas la seule scène emblématique de l'imaginaire ducharmien. On peut trouver d'autres séquences prégnantes, marquantes, facilement identifiables dans les scénarios, puis repérables dans les romans. Toutes ne sont pas aussi statiques. La scène de la toilette où le personnage-enfant traite le parent comme son propre enfant en le baignant, coiffant ou bichonnant réapparaît, elle aussi, non seule-

L'un(e) dort, l'autre pas: la scène de la veille dans les scénarios et quelques romans... 
ment dans tous les scénarios mais aussi, par exemple, dans Les Enfantômes. Elle concrétise, elle aussi, une inversion de rapports et de pouvoir entre parents et enfants. On peut aussi noter la récurrence des scènes de saut. Par ailleurs, il est bien d'autres aspects communs entre les romans et les scénarios: lieux, animaux, voitures, dialogues percutants, goût de la bohême, de l'excentricité qui frappe encore bien davantage dans des textes destinés à l'écran. En privilégiant la présentation scénique, j'espère cependant avoir touché à un aspect clé de l'art du scénario.

\section{L'art du scénario}

D'après la méthode critique de Charles Mauron, on pourrait, à partir de l'interprétation de la scène de la veille, tenter d'entreprendre une exploration de l'inconscient du romancier-scénariste. Il faudrait, suivant l'exemple de Mauron, construire, à partir d'autres scènes, un modèle qui permette de cerner le mythe personnel de l'écrivain. L'œuvre de Ducharme offre, sans aucun doute, un terrain fertile à la psychocritique ${ }^{7}$. Cependant, plutôt que de poursuivre une recherche déjà très riche ${ }^{8}$, je préfere amorcer une réflexion critique sur l'art du scénario.

L'analyse de Mauron conduit à des généralisations qui découlent d'un processus d'abstraction. À son stade ultime, le schéma de la configuration des rapports entre personnages est complètement détaché des situations concrètes dont il a été dérivé. Si cette démarche permet de "dénuder l'inconscient de l'écrivain", elle a l'inconvénient d'effacer ce qui constitue un aspect essentiel de l'art du scénariste et aussi, bien que dans une moindre mesure, du romancier. La scène de la veille est un chronotope important de l'imaginaire ducharmien dans le sens que lui donne Bakhtine: "un centre de concrétisation figurative, à caractère sensuellement concret". "Dans le chronotope, dit le critique russe, les événements du roman prennent corps et se revêtent de chair, s'emplissent de sang" (p. 391). Ce qui me fascine dans ces scènes emblématiques, c'est tout autant une configuration de rapports de forces, le faible prenant sa revanche sur le fort, que leur actualisation: le sommeil de l'un, le regard de l'autre, l'araignée de l'une, la grenouille de l'autre, le linceul, les 
fleurs, le "Barbie" masculin, l'ours en peluche, la présence des corps, sans oublier la musique. Le désir d'aimer et de dominer l'autre se revêt de chair visuellement et auditivement à travers des situations, des gestes, des regards, des objets...

Lart du scénariste se manifeste à travers le pouvoir évocateur de cette figuration, non seulement par rapport à la situation des personnages, mais aussi par rapport à l'écho qu'elle peut susciter chez le spectateur. En effet, le talent du scénariste ne se limite pas à inventer une histoire bien construite, mise en dialogues appropriés, mais aussi surtout à la concrétiser dans l'espace et le temps diégétiques, à travers des situations, des activités spécifiques, riches en connotations et d'autant plus frappantes pour le public qu'elles sont proches du quotidien. La scène de la veille constitue un exemple privilégié de cette concrétisation. Même s'il a peu de chances de trouver des producteurs à Hollywood, Ducharme n'en est pas moins, à mon avis, un excellent scénariste parce qu'il possède l'art de créer de telles situations.

Un des poncifs de la critique de scénarios affirme que tout bon scénariste doit "écrire visuellement ${ }^{9}$ ", que la lecture du texte doit immédiatement suggérer des images. Il est évident que, dès le stade de la continuité, tout scénario doit se composer de scènes propres à l'expression cinématographique. Il est plus difficile, mais aussi plus intéressant, de chercher à caractériser la "visualité" propre au scénario. Comme le montrent les extraits déjà cités, les passages narrativo-descriptifs du scénario sont bien moins riches en détails visuels que, par exemple, les descriptions du roman réaliste. Une telle précision ne se justifie pas dans un scénario. C'est la réalité filmée qui concrétisera le contenu des images. Les matrices visuelles du scénario fonctionnent différemment des descriptions romanesques. Elles peuvent se dispenser des détails visant "l'effet de réel ». Elles se fondent avant tout sur des mises en scène, des mises en situation, des actions fictionnelles.

Si la scène de la veille est profondément intégrée dans l'imaginaire ducharmien parce qu'elle concrétise des rapports affectifs fondamentaux entre les personnages, elle convient aussi particulièrement à une représentation cinématographique grâce à une convergence entre cette mise en rapport des personnages et le 
médium filmique. C'est qu'en effet, elle se focalise suivant le regard sur l'autre, être désiré qui, endormi, s'abandonne enfin à la contemplation. On sait l'importance, dans la critique cinématographique, de la réflexion sur le regard, le fameux gaze anglais, qui caractérise non seulement la situation du personnage par rapport au dormeur (identification secondaire), mais aussi celle du spectateur ou de la spectatrice par rapport au personnage (identification primaire ${ }^{10}$ ). Le cinéma magnifie le regard du spectateur et abolit le regard de l'autre (celui du personnage). Les scènes de veille imaginées par Ducharme soulignent le pouvoir érotique du corps contemplé par ce double regard. Il est d'autres scènes de veille mémorables au cinéma, par exemple, celle où la Bête contemple Belle endormie, dans le film de Jean Cocteau.

L'épreuve de force pour un scénario se situe à l'étape de la réalisation. Le potentiel cinématographique du texte ne dépend pas de l'abondance des directives techniques. Tout le monde s'accorde à présent pour laisser la responsabilité de la planification des images au réalisateur, au moment de l'élaboration du découpage technique. Les scénarios de Ducharme, comme la majorité des scénarios actuels, ne spécifient pas les modalités de prises de vues. Leurs qualités cinématographiques tiennent, entre autres, à l'invention de situations, telle la scène de la veille, permettant de concrétiser les rapports entre personnages. Il n'est pas question d'analyser ici les films réalisés par Francis Mankiewicz ${ }^{11}$, mais on s'en voudrait de passer sous silence cette lecture extraordinairement sensible des scénarios de Ducharme, par un réalisateur qui connaissait les romans et s'est laissé prendre à ce monde unique, si évocateur de la personnalité de son créateur: "C'est une écriture dramatique [celle de Ducharme] quasiment pure. Elle devient elle-même un des éléments du film. De sorte que le film ne soit pas une reproduction mais une interprétation de la vie ${ }^{12}$." Les scènes de veille des deux films sont filmées avec une sensibilité qui transmet toute leur intensité émotive. Dans Les Beaux Souvenirs, par exemple, la mise en scène joue habilement de l'ombre et de la lumière du jour naissant. Marie, merveilleusement jouée par Monique Spaziani, se berce sur une chaise tout en contemplant son père endormi, évoquant, par ce 
mouvement, toute la jouissance de la veille. Le plan rapproché de Manon et de Michelle, à la fin des Bons Débarras, que j'ai choisi pour illustrer cet article, communique bien, lui aussi, toute la charge affective de ce moment.

On pourrait poursuivre cette comparaison entre scénarios et films. Mais, ce n'est pas l'objectif de cet article. Mon but était de montrer combien les scénarios sont révélateurs de l'imaginaire d'un auteur. Ce monde imaginaire se compose de mythes personnels obsédants qui se révèlent à travers une fascination certaine pour des situations spécifiques, focalisées sur des rapports corporels représentant des liens affectifs entre personnages. Si la scène de la veille occupe une place centrale dans les scénarios et seulement mineure dans les romans, c'est sans doute à cause de sa convergence particulière avec le médium filmique.

De plus, il me semble que l'analyse des scénarios permet de jeter une lumière quelque peu nouvelle sur l'œuvre romanesque. Des études critiques extrêmement intéressantes, celles de PierreLouis Vaillancourt ou d'Anne-Élaine Cliche, présentent les romans de Ducharme uniquement comme des "mises en scène du langage et de l'écriture ", des "fêtes de mots, folie de paroles" et vont jusqu'à y voir " une absence généralisée d'actions fictionnelles ". Est-ce habitude de lecture des scénarios? Pour ma part, je ne peux m'empêcher d'être frappée, surtout dans les romans récents, par la force dramatique de certaines scènes. Ainsi, à la fin de Dévadé, les sept pas "bouddhiques" de la patronne qui vient s'étaler, face contre terre, aux pieds de Bottom. Cette scène-là pourrait, elle aussi, figurer dans un scénario.

Simon Fraser University

\section{NOTES}

1 Version remaniée et augmentée d'une communication présentée au colloque de l'APFUCC, Ottawa, congrès des Sociétés savantes, mai 1993.

2 Nous parlons ici des "scénarios d'auteur ", comme on dit "cinéma d'auteur", qui portent la marque de leur créateur. Tous les scénarios ne sont pas ainsi.

3 Le Nez qui voque, adapté à l'écran par le metteur en scène français Alain Périsson, production franco-canadienne, a été tourné en 1971. Selon Périsson, Ducharme aurait collaboré aux premiers stades de la scénarisation, mais la participation du romancier n'est pas reconnue dans le générique du film.

L'un(e) dort, l'autre pas : la scène de la veille dans les scénarios et quelques romans... 
4 Je tiens à remercier Nicole Laurin d'avoir mis ces textes à ma disposition. Ce sont ces textes, conservés à la Cinémathèque québécoise, qui nous serviront de référence tout au long de cet article.

5 Les scénarios de Ducharme ont déjà fait l'objet d'une étude générale intéressante. Il est significatif que celle-ci ait paru dans un numéro thématique sur les rapports entre littérature et cinéma: Diane Pavlovic, "Réjean Ducharme scénariste: les p(l)ans de l'illusion ", Revue d'histoire littéraire du Québec, nº 11 (1986) p. 85-92.

6 Cette caractérisation des personnages vient directement de Ducharme qui fait leur portrait moral au début de chaque scénario.

7 Je suppose qu'on pourrait, par exemple, à propos de la scène de la veille, évoquer la fameuse scène primitive.

8 Voir par exemple, l'étude d'Anne-Élaine Cliche, Le Désir du roman. Hubert Aquin, Réjean Ducharme (Montréal: XYZ, 1992).

9 Voir Jacqueline Viswanathan, "Le discours sur l'image", Semiotica, vol. 40, $\mathrm{n}^{\circ} 1 / 2(1982)$ p. $27-43$ et "Une histoire racontée en images", Etudes françaises, 22/3 (hiver 1987), p. 72-81.

10 Voir à ce sujet, J. Aumont, A. Bergala, M. Marie et M. Vernet, Esthétique du film (Paris: Nathan, 1983), notamment le sous-chapitre, "La double identification au cinéma".

11 D'après une entrevue avec Francis Mankiewicz, c'est Réjean Ducharme qui, après avoir contacté plusieurs personnes dont Jacques Godbout, a finalement réussi à obtenir l'adresse du réalisateur pour lui envoyer la première version du scénario des Bons Débarras. Ducharme avait beaucoup admiré le premier film de Mankiewicz, Le Temps d'une chasse. Ducharme a retravaillé une première fois le scénario d'après les suggestions du réalisateur. Puis, ils se sont rencontrés pour rédiger ensemble la dernière version. Ducharme n'est pas intervenu pendant le tournage. Voir: "To Berlin with Love: an Interview with Francis Mankiewicz by Joan Irving", Cinema Canada (mars 1980) p. 11-15.

12 Voir "Entrevue de Jean Royer avec Francis Mankiewicz", Le Devoir (7 novembre, 1981).

\section{BIBLIOGRAPHIE}

Bakhtine, Mikhaïl. Esthétique et théorie du roman. Paris: Gallimard, 1978.

Clerc, Jeanne-Marie. Ecrivains et cinéma. Des mots aux images et des images aux mots: adaptations et ciné-romans. Paris/Metz: Klincksieck/ Presses universitaires de Metz, 1985.

Cliche, Anne-Élaine. Le Désir du roman. Hubert Aquin. Réjeah Ducharme. Montréal: XYZ, 1992.

Cohen, Keith. Film and Fiction/The Dynamics of Exchange. New Haven/London: Yale University Press, 1979.

Ducharme, Réjean. Dévadé. Paris: Gallimard, 1990.

Ducharme, Réjean. L'Avalée des avalés. Paris: Gallimard, 1966.

Ducharme, Réjean. L'Hiver de force. Paris: Gallimard, 1973.

Ducharme, Réjean. Le Nez qui voque. Paris: Gallimard, 1967.

Ducharme, Réjean. Les Enfantômes. Paris: Gallimard, 1976.

Gaudreault, André. Du Littéraire au filmique. Système du récit. Paris: Méridiens / Klincksieck, 1988.

Gaudreault, André et Jost, François. Le Récit cinématographique. Paris : Nathan, 1990. 
Jost, François. L'Eil-caméra. Entre film et roman. Lyon: Presses Universitaires de Lyon, 1987.

Magny, Claude-Edmonde. L'Âge du roman américain. Paris: Seuil, 1948.

Mauron, Charles. Des Métaphores obsédantes au mythe personnel. Introduction à la psychocritique. Paris: José Corti, 1962.

Poulet, George. L'Espace proustien. Paris: Gallimard, 1963.

Richard, Jean-Pierre. Littérature et sensation. Paris: Seuil, 1954.

Ropars-Wuilleumier, Marie-Claire. De la Littérature au cinéma. Genèse d'une écriture. Paris: Armand Colin, 1970.

Vaillancourt, Pierre-Louis. "L'offensive Du-Charme». Voix et Images, vol. V (automne 1979) p. 177-185.

Vaillancourt, Pierre-Louis. "Sémiologie de l'ironie: l'exemple de Ducharme». Voix et Images, vol. VII, $\mathrm{n}^{\circ} 3$ (1982) p. 513-522.

Vanoye, Francis. Récit écrit, récit filmique. Paris : Nathan, 1989.

L'un(e) dort, l'autre pas : la scène de la veille dans les scénarios et quelques romans... 207 